\title{
gु \\ Theory of Raman response of a superconductor with extended $s$-wave symmetry: Application to the iron pnictides
}

\author{
Andrey V. Chubukov, ${ }^{1}$ Ilya Eremin, $, 2,3$ and Maxim M. Korshunov 2,4 \\ ${ }^{1}$ Department of Physics, University of Wisconsin-Madison, Madison, Wisconsin 53706, USA \\ ${ }^{2}$ Max-Planck-Institut für Physik komplexer Systeme, D-01187 Dresden, Germany \\ ${ }^{3}$ Institute für Mathematische und Theoretische Physik, TU Braunschweig, D-38106 Braunschweig, Germany \\ ${ }^{4}$ L. V. Kirensky Institute of Physics, Siberian Branch of Russian Academy of Sciences, 660036 Krasnoyarsk, Russia
}

(Received 14 January 2009; published 1 June 2009)

\begin{abstract}
We argue that Raman study of Fe-pnictides is a way to unambiguously distinguish between various superconducting gaps proposed for these materials. We show that $A_{1 g}$ Raman intensity develops a true resonance peak below $2 \Delta$ if the pairing gap has $A_{1 g}$ symmetry in the folded Brillouin zone $(\Delta(k=0)=\Delta, \Delta(\pi, \pi)=-\Delta)$. No such peak develops for a pure $s$-wave gap, a $d$-wave gap, and an extended $s$-wave gap with $\Delta(\mathbf{k})$ $=\Delta \cos \frac{k_{x}}{2} \cos \frac{k_{y}}{2}$. We show that the peak remains quite strong for the values of interpocket impurity scattering used to fit NMR data.
\end{abstract}

DOI: 10.1103/PhysRevB.79.220501

PACS number(s): 74.20.Mn, 74.20.Rp, 74.25.Jb, 74.25.Gz

Recent discovery of superconductivity (SC) in the ironbased layered pnictides with $T_{c}$ reaching $55 \mathrm{~K}$ generated enormous interest in the physics of these materials. ${ }^{1}$ Most of ferropnictides are quasi-two-dimensional materials, and their parent (undoped) compounds are metals and display antiferromagnetic long-range order below $T_{N} \sim 150 \mathrm{~K} .{ }^{1-3}$ Superconductivity occurs upon doping of either electrons or holes into FeAs layers or by applying pressure. The electronic structure measured by angle-resolved photoemission (ARPES) (Ref. 4) and by magneto-oscillations ${ }^{5}$ consists of two small hole pockets centered around the $\Gamma=(0,0)$ point and two small electron pockets centered around the $M$ $=(\pi, \pi)$ point in the folded Brillouin zone (BZ). The sizes of electron and hole pockets are about equal in parent compounds.

The key unresolved issue for the pnictides is the symmetry of the superconducting gap. A conventional phononmediated $s$-wave superconductivity is unlikely because electron-phonon coupling calculated from first principles is quite small. ${ }^{6}$ Several authors considered magnetically mediated pairing based either on itinerant ${ }^{7-10}$ or localized spin models ${ }^{11}$ and argued that the gap should have an extended $s$-wave symmetry $\cos k_{x}+\cos k_{y}$ (also called $s^{+}$or, equivalently, $A_{1 g}$ symmetry). This gap changes sign between hole and electron pockets but has no nodes along the Fermi surface (FS). On the other hand, another random-phase approximation (RPA) study of magnetically mediated superconductivity in the five-band Hubbard model ${ }^{12}$ yielded two nearly degenerate candidate states in which the gap has nodes on one of the FS sheets: either an extended $s$-wave state with $\Delta(\mathbf{k}) \approx \Delta \cos \frac{k_{x}}{2} \cos \frac{k_{y}}{2} \quad$ or $\quad$ a $\quad d_{x^{2}-y^{2}}$ state with $\Delta(\mathbf{k})$ $\approx \Delta \sin \frac{k_{x}}{2} \sin \frac{k_{y}^{2}}{2}$ (in the unfolded BZ, these two states are $\cos q_{x}+\cos q_{y}$ and $\cos q_{x}-\cos q_{y}$, respectively ${ }^{13}$ ).

The experimental situation is also controversial. ARPES (Refs. 14 and 15) and Andreev spectroscopy ${ }^{16}$ measurements have been interpreted as evidence for a nodeless gap, either pure $s$ wave or $s^{+}$wave. The resonance observed in neutron measurements ${ }^{17}$ is consistent with the $s^{+}$gap. ${ }^{18}$ On the other hand, nuclear magnetic resonance (NMR) data ${ }^{19}$ and some of penetration depth data ${ }^{20}$ were interpreted as evidence for the nodes in the gap. Some but not all of the data can be reasonably fitted by a model of an $s^{+}$SC with ordinary impurities. ${ }^{21-23}$

In view of both theoretical and experimental uncertainties, it is important to find measurements which could unambiguously distinguish between different pairing symmetries. Recent suggestions for such probes include Andreev bound state $^{24}$ and Josephson interferometry. ${ }^{25}$ In this communication, we argue that the study of $A_{1 g}$ Raman intensity is another way to determine the symmetry of the superconducting gap. We show that in the $A_{1 g}$ scattering geometry the Raman signal develops a true resonance below $2 \Delta$ for the case of $s^{+}$ gap. No such resonance appears for a pure $s$-wave gap, for $\cos \frac{k_{x}}{2} \cos \frac{k_{y}}{2}$, and for $\sin \frac{k_{x}}{2} \sin \frac{k_{y}}{2}$ gaps. The $A_{1 g}$ resonance is the effect of the final state interaction, which is known to be important for Raman scattering. ${ }^{26} \mathrm{~A}$ similar resonance occurs in the $B_{1 g}$ channel in a magnetically mediated $d_{x^{2}-y^{2}}$ superconductor, ${ }^{27}$ but there the resonance is weakened by a finite damping associated with nodes of the $d$-wave gap.

We model Fe-pnictides by an itinerant electron system with two (almost) degenerate hole FS pockets centered at the $\Gamma$ point and two electron FS pockets centered at the $M$ point. We assume that the magnitude of the gap $\Delta$ is much smaller than the Fermi energy. In this situation, Raman intensity at frequencies $\leq 2 \Delta$ is determined by states near the FS where the density of states (DOS) can be approximated by a constant. We first assume that the pairing gap has $s^{+}$symmetry, $\Delta(\mathbf{k} \approx 0)=\Delta$ and $\Delta(\mathbf{k} \approx \pi)=-\Delta$, and show how the resonance appears. We then discuss other pairing symmetries.

Without final state interaction, the Raman intensity in a clean BCS $s^{+}$superconductor is the same as in a pure $s$-wave superconductor ${ }^{26}$ and is given by $I_{i}(\Omega)=2 \operatorname{Im} R_{i}(\Omega)$, where

$$
\begin{aligned}
R_{A_{1 g}}(\Omega)= & -R_{0}\left\langle\int d \omega \gamma_{A_{1 g}^{2}}^{2}\left[1-\frac{\omega_{+} \omega_{-}-\Delta^{2}}{\sqrt{\omega_{+}^{2}-\Delta^{2}} \sqrt{\omega_{-}^{2}-\Delta^{2}}}\right]\right. \\
& \left.\times \frac{1}{\sqrt{\omega_{+}^{2}-\Delta^{2}}+\sqrt{\omega_{-}^{2}-\Delta^{2}}}\right\rangle_{\mathrm{FS}} .
\end{aligned}
$$

Here $\gamma_{A_{1 g}}=\cos k_{x}+\cos k_{y}$ is the geometrical factor for $A_{1 g}$ 

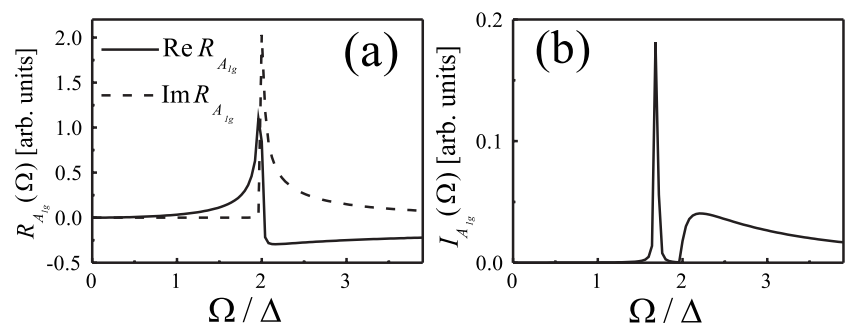

FIG. 1. Real and imaginary parts of the $A_{1 g}$ Raman intensity (in arbitrary units) for a clean $s^{+}$superconductor (a) without and (b) with final state interaction. Final state interaction gives rise to a well-defined resonance in the $A_{1 g}$ intensity. We used $u_{\mathrm{eff}} R_{0} \approx 0.4$ and added damping $\gamma=0.001 \Delta$.

scattering, $\omega_{ \pm}=\omega \pm \Omega / 2$, and $\langle\ldots\rangle_{\mathrm{FS}}$ denotes the averaging over FS. The factor 2 in the relation between $I_{i}(\Omega)$ and $R_{i}(\Omega)$ reflects the fact that there are two hole and two electron pockets. Other factors are incorporated into $R_{0}$. Like in Ref. 28 , we only consider the "low-energy" contribution to $R_{A_{1 g}}(\Omega)$ and neglect the constant "high-energy" term. This high-energy term does not affect $\operatorname{Im} R_{A_{1 g}}(\Omega)$ in Eq. (1) and also does not contribute to vertex renormalization if, as we assume, interactions begin to decay at energies smaller than the fermionic bandwidth (for a discussion, see Ref. 28).

The intensity $I_{A_{1 g}}(\Omega)$ computed using Eq. (1) vanishes at $\Omega<2 \Delta$ and is discontinuous at $2 \Delta$. The real part of $R_{A_{1 p}}$, which we will need later, is positive below $2 \Delta$, scales as $\Omega^{2}$ at small frequencies, and diverges upon approaching $2 \Delta$ from below. ${ }^{28}$ We show both $\operatorname{Re} R_{A_{1 g}}$ and $\operatorname{Im} R_{A_{1 g}}$ in Fig. 1. The final state interaction is diagrammatically represented as the renormalization of the Raman vertex. Vertex corrections arise from multiple insertions of fermion-fermion interactions into the Raman bubble. There are five different interactions between low-energy fermions [see Fig. 2(a)]. They include intraband interactions for electrons and for holes ( $u_{4}=u_{5}$ for particle-hole symmetric case which we consider), interband interactions $u_{1}$ and $u_{2}$ with momentum transfer 0 and $(\pi, \pi)$, respectively, and the pair hopping term $u_{3}$.

A generic theory of vertex renormalizations has been developed in Ref. 26, and we follow this work in our analysis. In general, there are three different types of vertex corrections: (i) the corrections which come from short-range interactions $u_{i}$ and transform a bare $A_{1 g}$ Raman vertex into a renormalized particle-hole vertex (these corrections involve $G G$ and $F F$ bubbles, where $G$ and $F$ are normal and anomalous Green's functions), (ii) the corrections which transform a Raman vertex into a particle-particle vertex (these involve $G F$ bubbles) [see Fig. 2(b)], and (iii) the corrections from the long-range component of the Coulomb interaction $V_{q} \propto 1 / q^{2}$. The corrections of the first type are given by ladder and bubble diagrams which involve the vertices $u_{1}, u_{2}$, and $u_{4}$ [first eight terms in Fig. 2(c)]. These are similar to the ones considered in Ref. 26, but in our case we need to include both ladder and bubble corrections; the latter is nonzero if $u_{1}$ is different from $u_{4}$. The corrections of the second type are nonzero when the symmetry of the gap is the same as the symmetry of the Raman vertex, which is our case. These corrections transform $A_{1 g}$ particle-hole vertex into an ordinary $s$-wave pairing vertex [the terms with the overall factor (a)

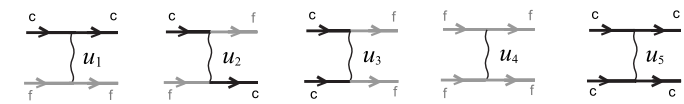

(b)

(c)
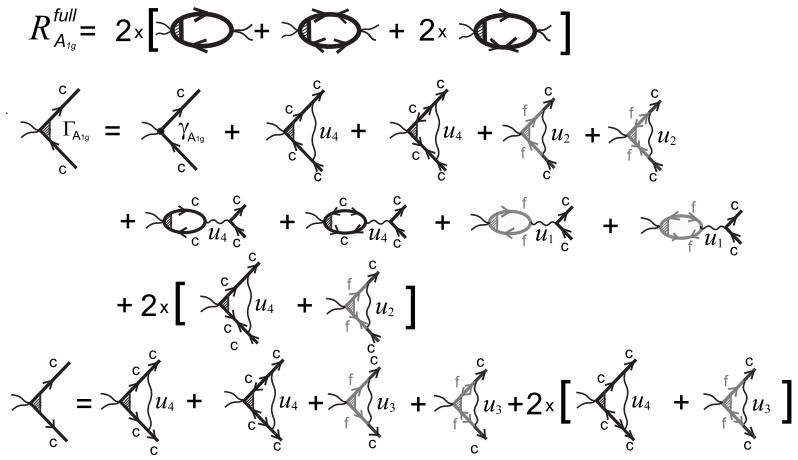

(d)

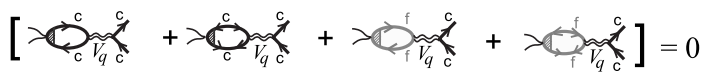

FIG. 2. (a) Five relevant interactions between fermions near hole and electron FS pockets. Black and gray lines represent fermionic $c$ states near $(0,0)$ and $f$ states near $M=(\pi, \pi)$. (b) The full Raman bubble, which is the sum of $G G, F F$, and $G F$ terms. Only the contribution from $c$ fermions is shown. The one from $f$ fermions is obtained by replacing $c$ lines by $f$ lines and vice versa in (b) and (c) panels. (c) The renormalization of the $A_{1 g}$ Raman vertex for $c$ fermions. The first eight diagrams account for "conventional" renormalization of the $A_{1 g}$ particle-hole vertex and involve $G G$ and $F F$ bubbles; the last two diagrams involve $G F$ bubbles and emerge due to a nonzero coupling between $A_{1 g}$ particle-hole channel and ordinary $s$-wave pairing channel. The renormalization of the particleparticle vertex in turn involves four conventional diagrams with $G G$ and $F F$ terms, which account for the renormalization in the particleparticle channel, and two diagrams due to the coupling to the $A_{1 g}$ particle-hole channel. (d) The renormalizations due to long-range component of Coulomb interaction $V_{q} \propto 1 / q^{2}$. This renormalization vanishes because of the symmetry between $c$ and $f$ fermions and the fact that $\gamma_{A_{1 g}}(k=0)=-\gamma_{A_{1 g}}(\pi)$.

2 in Fig. 2(c)]. The third renormalization, due to long-range component of the Coulomb interaction, generally gives rise to a screening of the Raman signal ${ }^{26,29}$ but vanishes in our case because of particle-hole symmetry and the fact that $A_{1 g}$ Raman vertex $\gamma_{A_{1,}}$ changes sign between hole and electron pockets [see Fig. $2(\mathrm{~d})$ ]. Note in this regard that $V_{q} \propto 1 / q^{2}$ is not a part of RG transformation and depends only on a momentum transfer $q$, in distinction to the other two interactions with small momentum transfer, $u_{4}$ and $u_{1}$. The bare values of $u_{4}$ and $u_{1}$ may be identical but the two flow in different directions under RG. Also note that we did not include a momentum-independent term into $\gamma_{A_{1 g}}$. If $\gamma_{A_{1 g}}$ had such component, it would be screened by the long-range Coulomb interaction.

Combining renormalizations (i) and (ii) and evaluating the diagrams, we obtain the full Raman intensity $I_{A_{1 g}}^{\text {full }}(\Omega)=2 \operatorname{Im} R_{A_{1 g}}^{\text {full }}(\Omega)$ with $R_{A_{1 g}}^{\text {full }}(\Omega)$ in the form

$$
R_{A_{1 g}}^{\text {full }}(\Omega)=\frac{R_{A_{1 g}}(\Omega)\left[1+u_{f} R_{\mathrm{pp}}(\Omega)\right]+4 u_{f} R_{\text {mix }}^{2}(\Omega)}{\left[1-u_{\mathrm{eff}} R_{A_{1 g}}(\Omega)\right]\left[1+u_{f} R_{\mathrm{pp}}(\Omega)\right]-4 u_{g} u_{f} R_{\mathrm{mix}}^{2}(\Omega)},
$$

where $u_{\text {eff }}=2 u_{1}-u_{2}-u_{4}$ is the effective vertex for the Raman renormalization in the $A_{1 g}$ particle-hole channel, $u_{f}=u_{3}+u_{4}$ 
and $u_{g}=u_{4}-u_{2}, R_{\mathrm{pp}}(\Omega) \propto \log E_{F} / \Omega>0$ is the polarization bubble in the $s$-wave particle-particle channel, and $R_{\text {mix }}(\Omega) \propto \int d^{2} k d \omega \gamma_{A_{1 g}} G_{k, \omega+\Omega} F_{k, \omega}$ couples $A_{1 g}$ particle-hole channel and $s$-wave particle-particle channel. At low frequencies, $R_{\text {mix }}(\Omega) \propto \Omega$. In this respect, the situation is similar to the case of a spin resonance in a $d$-wave superconductor, where $S=1$ particle-hole channel couples to $S=0$ particleparticle channel. ${ }^{30}$

Because $s$-wave channel is repulsive in our case $\left(u_{3}+u_{4}>0\right)$, there is no pole in $R_{A_{1 g}}^{\text {full }}(\Omega)$ coming from the particle-particle channel. Furthermore, $R_{\mathrm{pp}}(\Omega)$ logarithmically diverges at $\Omega \ll E_{F}$, and canceling this divergent term between the numerator and the denominator in Eq. (2), we obtain

$$
I_{A_{1 g}}^{\text {full }}(\Omega) \approx 2 \frac{\operatorname{Im} R_{A_{1 g}}}{\left(1-u_{\text {eff }} \operatorname{Re} R_{A_{1 g}}\right)^{2}+\left(u_{\text {eff }} \operatorname{Im} R_{A_{1 g}}\right)^{2}} .
$$

We see therefore that the coupling between $A_{1 g}$ particle-hole and $s$-wave particle-particle channels is irrelevant, and the full $I_{A_{1 g}}^{\text {full }}(\Omega)$ can be approximated by the expression which only includes vertex corrections which preserve particle-hole structure of the Raman vertex.

Our next observation is that for two-band structure, $u_{\text {eff }}$ contains the terms $u_{1}$ and $u_{2}$, which do not contribute to the renormalization of the $s^{+}$pairing vertex (the latter involves $u_{3}$ and $u_{4}$ terms $\left.^{21}\right)$, i.e., in distinction to one-band case, ${ }^{26}$ the renormalization of the $A_{1 g}$ Raman vertex and the renormalization of the $s^{+}$pairing vertex (which has the same $A_{1 g}$ symmetry) are given by different combinations of the interactions $u_{i}$

Finally, we note that below $2 \Delta, \operatorname{Im} R_{A_{1 g}}=0$ while $\operatorname{Re} R_{A_{1 g}}$ is positive and evolves between zero and infinity when $\Omega$ changes between zero and $2 \Delta$. Then, for positive $u_{\text {eff }}$, the $A_{1 g}$ Raman intensity develops a $\delta$-functional resonance peak below $2 \Delta$, at a frequency where $u_{\text {eff }} \operatorname{Re} R_{A_{1 g}}=1$. For a $d_{x^{2}-y^{2}}$ superconductor the same effect leads to an excitonic resonance in a staggered spin susceptibility ${ }^{31}$ and to a pseudoresonance in a $B_{1 g}$ Raman response. ${ }^{27}$

The flow of the interactions between the bandwidth $W$ and the Fermi energy $E_{F}$ has been analyzed in the earlier renormalization group (RG) study, ${ }^{21}$ and the result is that $u_{1}$ becomes the largest interaction at energies comparable to the Fermi energy, even if the intraband Hubbard repulsion $u_{4}$ is the largest term in the Hamiltonian. Specifically, in the RG flow $u_{1}$ and $u_{3}$ increase, $u_{2} / u_{1}$ flows to zero, and the $u_{4}$ term decreases such that $u_{\text {eff }}=2 u_{1}-u_{4}-u_{2}$ becomes positive at energies below $E_{F}$, relevant to Raman scattering, and the $A_{1 g}$ Raman response develops a resonance below $2 \Delta$. We emphasize that the physics which makes $u_{\text {eff }}$ positive is the same physics that gives rise to an attraction in an extended $s^{+}$-wave pairing channel. Indeed, the pairing interaction in $s^{+}$ channel, which is the combination $u_{3}-u_{4}$, becomes positive under RG.

For other proposed gap symmetries, the resonance does not develop even if one neglects the screening by long-range Coulomb interaction. For an $s$-wave gap, there is no sign change between electron and hole FS, and the analog of $u_{\text {eff }}$ in Eq. (3) is $-2 u_{1}-u_{4}+u_{2}$. This combination is negative, so
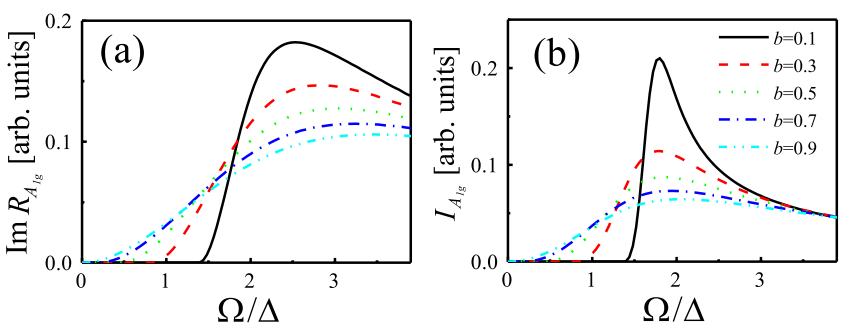

FIG. 3. (Color online) Calculated Raman intensity for an $s^{+}$ superconductor (a) without and (b) with final state interaction for various strength of the interband impurity scattering. We use the same $u_{\text {eff }}$ as in Fig. 1 and for definiteness set $U_{i}(0)=\Delta$.

the resonance does not occur. For $\Delta \propto \cos k_{x} / 2 \cos k_{y} / 2$ (s-wave gap with nodes), or $\Delta \propto \sin k_{x} / 2 \sin k_{y} / 2\left(d_{x^{2}-y^{2}}\right.$ gap), the gap changes sign along either hole or electron FSs. The largest contribution to $l_{A 1}(\Omega)$ comes from the FS along which the gap is nodeless, but vertex renormalization for such a term contains $u_{4}+\left(2 u_{1}-u_{2}\right) x$, where $x \sim k_{F}$ comes from the expansion of the gap around the FS where it has nodes. When $x \ll 1, u_{1}$ does not overcome $u_{4}$ and the resonance does not occur. For $d_{x y}$ gap 9 with $\Delta \propto \sin k_{x} \sin k_{y}$ (in the folded BZ), all $u_{i}$ terms in the vertex renormalization are reduced. Resonance may still occur, but the effective interaction now is small, $O\left(k_{F}^{2}\right)$, and the resonance is washed out by a small damping. This shows that the $A_{1 g}$ Raman resonance is a fingerprint of an $s^{+}$pairing.

Finally, we consider how the resonance in $s^{+}$superconductor is affected by ordinary impurities. As in earlier works, ${ }^{21,23}$ we introduce impurity potential $U_{i}(q)$ with intraand interpocket terms $U_{i}(0)$ and $U_{i}(\pi)$, respectively, and restrict with the Born approximation. This approximation (which requires $U_{i} \ll E_{F}$ ) may not work for $U_{i}(0)$ (Ref. 22) but should be valid for $U_{i}(\pi)$ which is pair breaking and is therefore very likely not larger than $\Delta \ll E_{F}$. For our case, $U_{i}(0)$ controls the functional form of $\operatorname{Re} R_{A_{1 g}}$, which still evolves between zero and infinity when $\Omega$ changes between 0 and $2 \Delta$, while the broadening of the resonance is entirely due to $U_{i}(\pi)$. In this situation, Born approximation should be sufficient.

The calculations are straightforward, and we refrain from presenting the details. Intrapocket impurity scattering does not affect the gap by Anderson's theorem, but $U_{i}(\pi)$, which scatter fermions with $+\Delta$ and $-\Delta$, is pair breaking and affects the gap in the same way as magnetic impurities in an ordinary $s$-wave superconductor. We use $b=2 U_{i}(\pi) / \Delta$, where $\Delta$ is the order parameter as a measure of the strength of pairbreaking impurity scattering.

The results of the calculations are shown in Fig. 3, where we plot Raman intensity in the presence of impurities both without and with final state interaction. Comparing this figure with Fig. 1 we see that the resonance gets damped at a finite $b$, and Raman intensity no longer shows two peaks. Still, the resonance continue to determine the shape of $I_{A_{1 g}}(\Omega)$ : without final state interaction the peak broadens and shifts to larger frequencies $\Omega>2 \Delta$ upon increasing $b$; while when the final state interaction is included, the peak remains below $2 \Delta$ and shifts to a smaller frequency with increasing $b$. 
Notice that the resonance is still quite strong at $b \sim 0.5-0.7$, which was used to fit NMR and penetration depth data. ${ }^{21,23}$ In other words, it should be observable in Raman experiments if indeed the gap has an $s^{+}$symmetry.

To conclude, in this Rapid Communication we argued that Raman study of Fe-pnictides is a way to unambiguously distinguish between various superconducting gaps proposed for these materials. We have shown that for an $A_{1 g}\left(s^{+}\right)$gap $\Delta(\mathbf{k} \approx 0)=\Delta, \Delta(\mathbf{k} \approx \pi) \approx-\Delta$, the $A_{1 g}$ Raman intensity has a true resonance peak below $2 \Delta$. No such peak emerges for a pure $s$-wave gap, a $d_{x^{2}-y^{2}}$ gap, and an extended $s$-wave gap with $\Delta(\mathbf{k})=\Delta \cos \frac{k_{x}}{2} \cos \frac{k_{y}}{2}$. The resonance peak gets broader by pair-breaking interpocket impurity scattering but is still fairly visible for the values of impurity scattering used to fit NMR data.

We acknowledge useful conversations with G. Blumberg, W. Brenig, H.-Y. Choi, D. V. Efremov, A. Sacuto, M. Vavilov, and A. Vorontsov. A.V.C. acknowledges support from NSF (Contract No. NSF-DMR 0604406). I.E. acknowledges partial support from the Asian-Pacific Center for Theoretical Physics, the Volkswagen Foundation (Contract No. I/82203), and the RMHE Program "Development of scientific potential" (Contract No. N1 2.1.1/298.5). M.M.K. acknowledges support from RFBR (Grant No. 09-02-00127) and RAS program on "Low temperature quantum phenomena."
*Also at Physics Department, Kazan State University, 420008 Kazan, Russian Federation.

${ }^{1}$ Y. Kamihara et al., J. Am. Chem. Soc. 130, 3296 (2008).

${ }^{2}$ Clarina de la Cruz et al., Nature (London) 453, 899 (2008).

${ }^{3}$ H. H. Klauss et al., Phys. Rev. Lett. 101, 077005 (2008).

${ }^{4}$ C. Liu et al., Phys. Rev. Lett. 101, 177005 (2008); D. V. Evtushinsky et al., Phys. Rev. B 79, 054517 (2009); D. Hsieh et al., arXiv:0812.2289 (unpublished); H. Ding et al., arXiv:0812.0534 (unpublished).

${ }^{5}$ A. I. Coldea et al., Phys. Rev. Lett. 101, 216402 (2008).

${ }^{6}$ L. Boeri et al., Phys. Rev. Lett. 101, 026403 (2008).

${ }^{7}$ I. I. Mazin et al., Phys. Rev. Lett. 101, 057003 (2008).

${ }^{8}$ Y. Yanagi et al., J. Phys. Soc. Jpn. 77, 123701 (2008); H. Ikeda, ibid. 77, 123707 (2008).

${ }^{9}$ Z.-J. Yao et al., New J. Phys. 11, 025009 (2009).

${ }^{10}$ F. Wang et al., Phys. Rev. Lett. 102, 047005 (2009).

${ }^{11}$ M. M. Parish et al., Phys. Rev. B 78, 144514 (2008).

${ }^{12}$ S. Graser et al., New J. Phys. 11, 025016 (2009).

${ }^{13}$ Strictly speaking, the extended $s$-wave states with $\Delta(\mathbf{k}) \propto \cos k_{x}$ $+\cos k_{y}$ and $\Delta(\mathbf{k}) \propto \cos \frac{k_{x}}{2} \cos \frac{k_{y}}{2}$ cannot be decoupled in the gap equation as both are members of the $A_{1 g}$ representation of $D_{4 h}$ tetragonal space group. However, for small hole and electron pockets, the matrix element between the two is small.

${ }^{14}$ T. Kondo et al., Phys. Rev. Lett. 101, 147003 (2008).

${ }^{15}$ H. Ding et al., Europhys. Lett. 83, 47001 (2008).

${ }^{16}$ T. Y. Chen et al., Nature (London) 453, 1224 (2008).

${ }^{17}$ A. D. Christianson et al., Nature (London) 456, 930 (2008); M. D. Lumsden et al., Phys. Rev. Lett. 102, 107005 (2009).

${ }^{18}$ M. M. Korshunov and I. Eremin, Phys. Rev. B 78, 140509(R) (2008); T. A. Maier and D. J. Scalapino, ibid. 78, 020514(R)
(2008).

${ }^{19}$ Y. Nakai et al., J. Phys. Soc. Jpn. 77, 073701 (2008); H.-J. Grafe et al., Phys. Rev. Lett. 101, 047003 (2008).

${ }^{20}$ R. T. Gordon et al., Phys. Rev. Lett. 102, 127004 (2009); R. T. Gordon et al., Phys. Rev. B 79, 100506(R) (2009); J. D. Fletcher et al., arXiv:0812.3858 (unpbulished).

${ }^{21}$ A. V. Chubukov et al., Phys. Rev. B 78, 134512 (2008).

${ }^{22}$ D. Parker et al., Phys. Rev. B 78, 134524 (2008).

${ }^{23}$ Y. Bang et al., Phys. Rev. B 79, 054529 (2009); Y. Senga and H. Kontani, J. Phys. Soc. Jpn. 77, 113710 (2008); A. B. Vorontsov et al., Phys. Rev. B 79, 060508(R) (2009).

${ }^{24}$ P. Ghaemi et al., Phys. Rev. Lett. 102, 157002 (2009).

${ }^{25}$ D. Parker and I. Mazin, arXiv:0812.4416 (unpublished); J. Wu and Ph. Phillips, Phys. Rev. B 79, 092502 (2009).

${ }^{26}$ M. V. Klein and S. B. Dierker, Phys. Rev. B 29, 4976 (1984); See also W.-C. Wu and A. Griffin, Phys. Rev. B 52, 7742 (1995); T. P. Devereaux and D. Einzel, ibid. 51, 16336 (1995); T. Strohm and M. Cardona, ibid. 55, 12725 (1997).

${ }^{27}$ A. Chubukov et al., Solid State Commun. 112, 183 (1999); A. V. Chubukov et al., Phys. Rev. B 73, 094512 (2006).

${ }^{28}$ A. V. Chubukov and M. R. Norman, Phys. Rev. B 77, 214529 (2008) Note that in this paper the irreducible vertex $\Gamma_{\alpha \beta, \gamma \delta}$ has opposite sign compared to our interaction potentials $u_{i}$.

${ }^{29}$ A. Abrikosov and V. M. Genkin, Zh. Eksp. Teor. Fiz. 65, 842 (1973) [Sov. Phys. JETP 38, 417 (1974)].

${ }^{30}$ O. Tchernyshyov et al., Phys. Rev. B 63, 144507 (2001); W. C. Lee et al., ibid. 77, 214518 (2008); Z. Hao and A. Chubukov, arXiv:0812.2697 (unpublished).

${ }^{31}$ See, e.g., M. Eschrig, Adv. Phys. 55, 47 (2006). 